\title{
Kein optimaler Behandlungserfolg ohne Therapiemanagement beim mRCC
}

\begin{abstract}
— Zielgerichtete Therapien haben die Behandlung des metastasierten Nierenzellkarzinoms (mRCC) revolutioniert, wie beispielsweise bei gutem/intermediärem Risiko Sunitinib (Sutent ${ }^{\oplus}$ ) oder bei hohem Risiko Temsirolimus (Torisel ${ }^{\circledR}$ ). Ein optimaler Therapieerfolg lässt sich laut Markus Kuczyk, Hannover, jedoch nur mit einem Therapiemanagement erreichen, das auf eine optimale Dosierung, eine möglichst lange Therapiedauer und ein individuelles Nebenwirkungsmanagement abzielt. Die Empfehlung, zielgerichtete Therapien so lange wie möglich einzusetzen, basiert auf der Erfahrung, dass das beste Ansprechen auf Sunitinib häufig erst nach langer Behandlung erreicht wird. Viktor Grünwald, Hannover, schilderte den Fall einer Patientin, welche die best response, eine sehr gute partielle Remission, erst nach 16,3 Monaten erreichte. Eine optimale Dosierung ist erforderlich, weil zwischen dem Ansprechen auf Sunitinib und der Exposition eine lineare Korrelation besteht.
\end{abstract}

Das Ziel einer möglichst langen Therapie können Nebenwirkungen gefährden. Ein individuelles Nebenwirkungsmanagement ist daher nach Einschätzung von Axel S. Merseburger, Hannover, unerlässlich. Erforderlich sind ein an Anamnese und klinische Befunderhebung adaptiertes prophylaktisches Nebenwirkungsmanagement. Die Patienten müssen aufgeklärt und intensiv überwacht werden. Auch leichte Nebenwirkungen sollten behandelt werden, um schwere zu vermeiden. Hilfreich ist laut Merseburger die jahrelange Erfahrung mit Sunitinib und Temsirolimus und die darauf basierende Expertise der Ärzte im Umgang mit den Nebenwirkungen.

Dr. Günter Springer

Fachjournalisten-Workshop „RCC: Potenziale von Sunitinib und Temsirolimus voll ausschöpfen: Therapiemanagement beim metastasierten Nierenzellkarzinom (mRCC)", Hannover, 2. Mai 2011

Veranstalter: Pfizer, Berlin

\section{Jahre Dr. Kade}

Die Firmengeschichte des Unternehmens Dr. Kade begann im Jahr 1886 damit, dass Dr. Franz Lutze die OranienApotheke seines Kollegen Dr. Rudolph Kade erwirbt. Innerhalb weniger Jahre entwickelte sich aus der Berliner Apotheke eine pharmazeutische Fabrik, die sowohl die deutschen Schutztruppen in den Kolonien beliefert als auch die Bord-Apotheke der kaiserlichen Yacht "Hohenzollern“. Hergestellt wurden damals unter anderem Verbandstoffe, Arzneien, Reiseapotheken und chirurgisches Gerät. Heute beschäftigt Dr. Kade über 400 Mitarbeiter an zwei Standorten in Deutschland. Mit dem Fokus auf die Bereiche Proktologie, Gynäkologie und Schmerztherapie ist das Unternehmen seinen Kernkompetenzen treu geblieben.

In den kommenden Jahren will das Unternehmen durch eigene Entwicklungsprojekte und durch Einlizenzierungen in den genannten Fachgebieten weiter wachsen.

Nach Informationen von

Dr. Kade, Berlin

\section{Der kleine Unterschied}

Männer und Frauen haben verschiedene Bedürfnisse - auch in punkto Blasenschwäche. Männer benötigen beispielsweise ein speziell an ihre Anatomie angepasstes Hilfsmittel. Frauen wünschen sich hingegen eher ein Produkt, das ihnen ein Gefühl von Weiblichkeit schenkt. Die neue Schutzunterwäsche von TENA berücksichtigt diese Unterschiede: Die TENA Men Protective Underwear Level 4 wurde speziell für die männlichen Bedürfnisse entwickelt. Und die TENA Protective Underwear mit ihrem femininen Design spricht die weibliche Klientel an. Beide Produkte bestehen aus einem baumwollähnlichen und weichen Material. Die eng anliegende Passform sorgt für Diskretion und ein angenehmes Tragegefühl. In das Produkt wurde ein besonders leistungsstarker Saugkern eingearbeitet. Das Männerprodukt verfügt zudem über einen verstärkten und verbreiterten Frontbereich. Unangenehme Gerüche werden durch den verbesserten $\mathrm{Ge}$ ruchsschutz Odour Control ${ }^{\mathrm{TM}}$ zuverlässig verhindert.

Mittagssymposium „Veregen ${ }^{\oplus} 10 \%$ Salbe Therapie von Genitalwarzen mit Extrakt aus grünem Tee", Dresden, 1. April 2011;

Veranstalter: Abbott Arzneimittel, Hannover
Nach Informationen von SCA hygiene Products, Mannheim 